\title{
Frozen Elephant Trunk with Gore Thoracic Endovascular Graft and Quadrifurcate Graft
}

\author{
Andrea Venturini ${ }^{1}$, Alan Gallingani ${ }^{2}$, Luca Zanella ${ }^{3}$, and Domenico Mangino ${ }^{1}$ \\ ${ }^{1}$ Ospedale dell' Angelo-Mestre \\ ${ }^{2}$ Ospedale Maggiore di Parma \\ ${ }^{3}$ University of Padua
}

April 28, 2021

\begin{abstract}
The frozen elephant trunk has simplified the surgical treatment of the entire thoracic aorta; moreover many different technical variants of how to do it have been described. Our procedure include implantation of a Gore CTAG graft into the aortic arch followed by a teflon reinforced suture line that should include the stent graft and the native aorta. The 4-branched graft is then sutured to the distal stump therefore creating a custom-made frozen elephant trunk hybrid prosthesis. Three patients with acute type A aortic dissection and primary entry tear in the arch were operated with this technique. There were no early or late deaths, moreover no neurologic events occurred. The purpose of this article is to introduce an innovative technique for total arch replacement with the combination of a Gore CTAG stent graft used as a frozen elephant trunk and a standard 4-branched graft for arch reconstruction.
\end{abstract}

Frozen Elephant Trunk with Gore Thoracic Endovascular Graft and Quadrifurcate Graft

Andrea Venturini MD, $\mathrm{PhD}^{1}$, Alan Gallingani $\mathrm{MD}^{1,2}$, Luca Zanella $\mathrm{MD}^{1,3}$, Domenico Mangino $\mathrm{MD}^{1}$.

${ }^{1}$ Cardiac Surgery Department, Ospedale dell'Angelo, Venice-Mestre, Italy.

${ }^{2}$ Cardiac Surgery Department, Ospedale Maggiore di Parma - Università di Parma, Parma, Italy.

${ }^{3}$ Cardiac Surgery Department, Università di Padova, Padova, Italy.

Running head: FET with Gore stent graft

Corresponding Author:

Alan Gallingani MD

Cardiac Surgery Department

Ospedale Maggiore - University of Parma

Via Gramsci 14, 43126 Parma, Italy

Phone: +39 0521703972 (office)

Fax: +390521 702188

Mobile: +393474910056

Email: agallingani@ao.pr.it

Data availability statement: anonymously available on justified request. 


\section{Funding: none.}

\section{Conflict of interest: none.}

Disclosures : none.

Patient consent statement: the patients gave his consent.

\section{Abstract}

The frozen elephant trunk has simplified the surgical treatment of the entire thoracic aorta; moreover, many different technical variants of how to do it have been described. Our procedure includes implantation of a Gore CTAG graft into the aortic arch followed by a teflon reinforced suture line that should include the stent graft and the native aorta. The 4-branched graft is then sutured to the distal stump therefore creating a custom-made frozen elephant trunk hybrid prosthesis.

Three patients with acute type A aortic dissection and primary entry tear in the arch were operated with this technique. There were no early or late deaths, moreover no neurologic events occurred. The purpose of this article is to introduce an innovative technique for total arch replacement with the combination of a Gore CTAG stent graft used as a frozen elephant trunk and a standard 4-branched graft for arch reconstruction.

Key words: frozen elephant trunk, aortic arch replacement, aortic dissection, aortic arch hybrid prosthesis.

\section{Introduction}

The purpose of this article is to introduce a how-to-do-it of total arch replacement with the combination of a CTAG (W. L. Gore \& Associates, Flagstaff, AZ) used as a frozen elephant trunk and a standard 4-branched Hemashield Platinum vascular graft (Maquet, Rastatt, Germany). The original elephant trunk technique has been first described by Borst in 1983 [1], furthermore in the early 2000s the Frozen Elephant trunk (FET) operation [2] has been introduced in order to treat in a single stage both the pathologies of the arch and descending thoracic aorta.

At the moment there are four hybrid ready-made FET prostheses: The E-Vita Open Plus (Jotec GmbH, Hechingen, Germany), the Thoraflex Hybrid (Vascutek Terumo, Inchinnan, Scotland, UK), the Cronus (MicroPort, Shanghai, China) and the Frozenix (Japan Lifeline, Tokyo, Japan). The first two above mentioned devices are mainly implanted in Europe, while the third and fourth one are available in China and Japan, respectively. In US and other countries where hybrid prostheses are not yet available, direct implantation of stent grafts is performed through the open aorta during circulatory arrest with or without graft-aorta suture fixation [3]. Other leading aortic teams advocate a two staged approach starting with debranching of the supra-aortic vessels and replacement of the ascending aorta, used as landing zone for a total endovascular second stage [4].

The essential of our procedure include implantation of Gore CTAG graft into the aortic arch followed by a teflon reinforced suture line that should include the stent graft and the native aorta so that all these structures are firmly joined together. This suture is usually performed between the innominate and the left carotid artery (zone 1). The 4-branched graft is then sutured to the previously prepared distal stump therefore creating a custom-made frozen elephant trunk hybrid prosthesis (Fig.1).

\section{Methods}

From December 2018 to December 2019, 3 patients were operated with this innovative technique at the "Ospedale dell'Angelo" in Venezia-Mestre (Italy). Preoperative CT scans showed an acute type A dissection with the primary entry tear in the arch for all patients. The extension of the dissection for patient 1 and 2 was from the sino-tubular junction (STJ) to the iliac vessels while for patient 3 was from the STJ to the proximal descending aorta. Patients were 55,54 and 77 years old respectively.

Arterial cannulation site was femoral in order to perfuse the lower body while performing the distal anastomosis. After institution of cardiopulmonary bypass (CPB) the aorta was cross-clamped and retrograde cold 
blood St Thomas cardioplegia was delivered. The ascending aorta was resected during cooling. Aortic valve replacement with a bioprostheses was performed only in the third patient.

Once $25^{\circ} \mathrm{C}$ naso-pharingeal temperature was reached, circulatory arrest and antegrade selective cerebral perfusion (ASCP) with True Flow RDB cannulas (Med Europe SRL, Bologna, Italy) placed in the innominate and left carotid arteries was initiated. The aorta was further resected till the origin of the left common carotid artery. The supra-aortic vessels were transected and their ostia were carefully sutured.

A $150 \mathrm{~mm}$ Gore CTAG was chosen without oversize if compared to the preoperative proximal descending thoracic aorta diameter. We routinely used angioscopy [5] during the procedure: hence the endoprosthesis was deployed into the true lumen under direct vision without a guidewire.

The stent graft was further fixed to the thoracic aorta in zone 1. Two large $(7-8 \mathrm{~mm})$ strips of teflon were used to reinforce this suture both internally and externally. At first a mattress $4 / 0$ polypropylene suture was performed, followed by a running suture line that should include teflon felts, stent graft and native aorta so that all these structures are firmly joined together (Fig.2).

Once distal reconstruction was completed, the 4-branched graft was anastomosed to the distal stump with a 4/0 polypropylene running suture and cannulated through his side branch for resumption of antegrade CPB. Secondly the third branch of the graft was anastomosed to the left subclavian artery. In the first patient the left subclavian was dissected and severely fragile, therefore the third branch was anastomosed end to side to the axillary artery. Rewarming was then started; subsequently the proximal anastomosis was carried out. Finally, arch reconstruction was completed reimplanting the left carotid and the innominate artery respectively.

Results

Cardiopulmonary bypass time were 187', 232' and 220' respectively. ASCP time were 100', 128' and 132' while hypothermic circulatory arrest time (with partial visceral perfusion) were 42', 54' and 45'. In all patients as soon as the stent graft was deployed a Foley catheter was inflated in the endoprosthesis and a low flow (1-2 liters per minute) perfusion through the femoral artery cannula was started in order to minimize the risks of visceral ischemia.

Intensive care unit stay was 8,7 and 7 days respectively; there were no early or late deaths, moreover no stroke or minor neurologic events occurred in the three patients. No renal failure was observed in our small cohort of patients.

Three months CT scan for patient 1 and 2 showed a persistent patency of the false lumen in the proximal descending aorta, therefore an additional endoprosthesis was inserted (Fig.3). One year follow-up confirmed total false lumen thrombosis. Three- and nine-months CT scan for the third patient showed a very satisfactory result.

\section{Conclusions}

The essential of our procedure include implantation of a Gore CTAG into the descending aorta, teflon reinforced suture of the graft to the native aortic arch usually in zone 1, total arch replacement with a 4branched vascular graft, antegrade selective cerebral perfusion and moderate hypothermic circulatory arrest.

For the time being, there is not a single FET hybrid prosthesis worldwide commercially available, moreover a wide selection of off-the-shelf Gore endovascular grafts are available in most cardiothoracic operating theaters. Our technique relies on the same endovascular skills necessary for the implantation of any FET hybrid prosthesis, therefore avoiding the learning curve of endovascular technology and the use of fluoroscopy at the time of the operation that would require a hybrid operating theater.

In addition, Dr Coselli underlined the versatility of the $\mathrm{Y}$ or double $\mathrm{Y}$ graft in contrast to the 4-branched graft for arch vessels reconstruction. The trifurcated graft may be tailored to each patient individual surgical findings, therefore being the first choice in many operations [6]. 
In conclusion we describe a technique that doesn't require a ready-made hybrid prosthesis; therefore, we can have an individually tailored approach for each patient, combining any possible stent graft with the appropriate vascular graft, leaving the surgeon free to use his preferred total arch configuration and position of the distal anastomosis.

Although our small serie of patients consists of acute aortic dissection only, this technique could be applied to any other aortic arch pathologies, such as chronic dissections or aneurysms. Moreover, we believe that because of the individually tailored approach and easy availability of the necessary material this technique will be useful in many situations. Further investigation is necessary to confirm these favorable findings.

Author contribution:

Andrea Venturini MD PhD: collecting data, drafting the paper.

Alan Gallingani MD: collecting data, drafting the paper, image editing

Luca Zanella MD: image editing.

Domenico Mangino MD: resources.

All authors critically revised and approved final version.

\section{References}

1. Borst HG, Walterbusch G, Schaps D. Extensive aortic replacement using "elephant trunk" prosthesis. Thorac Cardiovasc Surg 1983;31:37-40.

2. Karck M, Chavan A, Hagl C, Friedrich H, Galanski M, Haverich A. The frozen elephant trunk technique: a new treatment for thoracic aortic aneurysms. J Thorac Cardiovasc Surg 2003;125:1550-3

3. Roselli EE, Soltesz EG, Mastracci T, Svensson LG, Lytle BW. Antegrade delivery of stent grafts to treat complex thoracic aortic disease. Ann Thorac Surg. 2010;90:539-46.

4. Kent WDT, Hereto EJ, Wong JK, Appoo JJ. Ascending, Total Arch, and Descending Thoracic Aortic Repair for Acute DeBakey Type I Aortic Dissection Without Circulatory Arrest. Ann Thorac Surg 2012;94:e59-61.

5. Tsagakis K. Angioscopy as a supplement to frozen elephant trunk treatment. Ann Cardiothorac Surg 2013;2:653-5.

6. 10.Ouzounian M, LeMaire SA, Coselli JS. Open Aortic Arch Repair: State-of-the-Art and Future Perspectives. Semin Thoracic Surg 2013; 25:107-115.

Figure Legends

Fig.1 FET with GORE CTAG. (A) Under moderate hypothermic circulatory arrest, the aortic arch was resected to zone 1 and antegrade selective cerebral perfusion was promptly instituted. Then the previously selected GORE CTAG was inserted into the true lumen of the aortic arch. (B) The stent graft was further fixed to the thoracic aorta in zone 1. Two large strips of teflon were used to reinforce this suture both internally and externally. At first a mattress 4/0 polypropylene suture was performed, followed by a running suture line that should include the teflon felts, the stent graft and the native aorta so that all these structures are firmly joined together. (C) Once distal reconstruction was completed, the 4-branched graft was anastomosed to the distal stump with a 4/0 polypropylene running suture. (D) Final result of total arch reconstruction.

Fig.2 Intra-operative details (A) View of the distal stump after its completion. An angled clamp is positioned on the left subclavian artery. ASCP cannulas are inserted in the innominate and left carotid artery. A Foley catheter is inflated in the endoprosthesis in order to start lower body perfusion through a femoral artery cannula. (B) Detail of the 4-layers distal stump. The CTAG is well deployed into the distal aortic arch. The mattress and the running suture lines incorporating the 4-layers (teflon-endoprosthesis-aorta-teflon) are clearly visibile.

Fig.3 Post-operative 3D CT-scan. (A) This picture clearly shows the zone 1 teflon-reinforced distal anastomosis. (B) Supra-aortic vessels reconstruction with left subclavian artery extra-anatomic revascularization 
is visible.
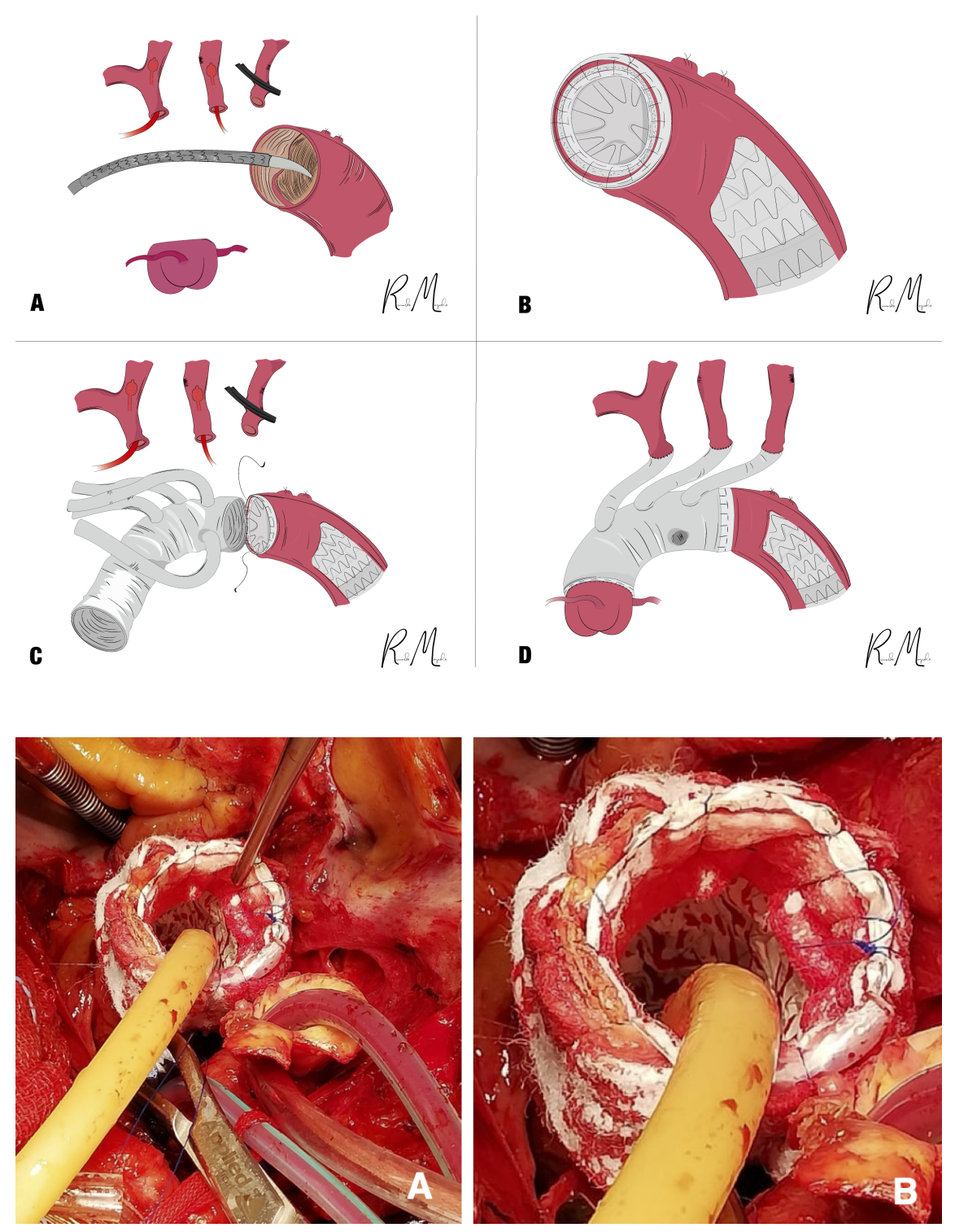


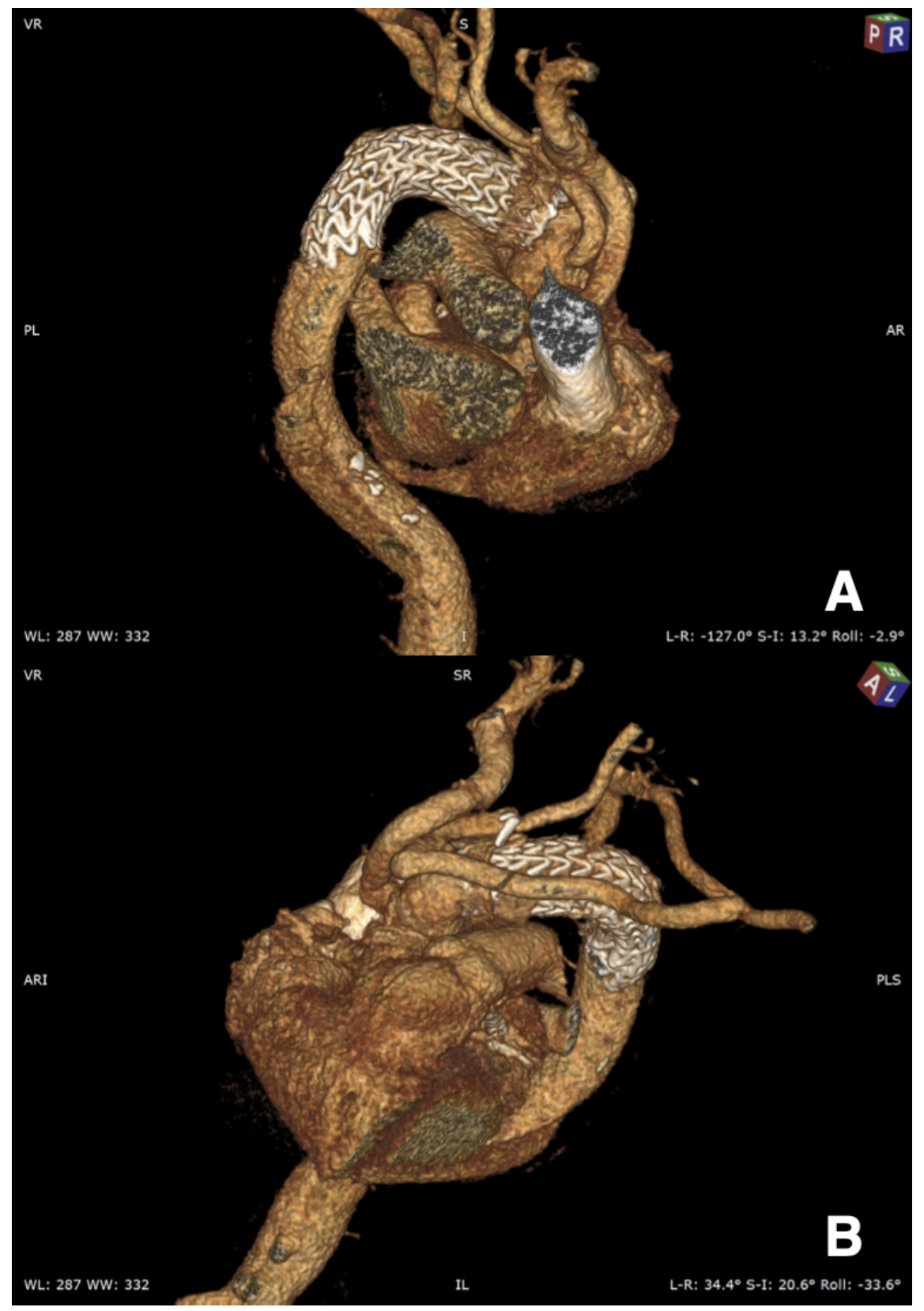

